\title{
ENERGY DISTRIBUTION OF Be STARS
}

\author{
GOPAL C. KILAMBI \\ Department of Astronomy, Osmania University, Hyderabad - 500 007, India
}

\section{Introduction}

Be stars are defined to be non-supergiant early-type stars of spectral type $B$ showing at times Balmer emission lines in their spectra. These stars often develop strong stellar winds considered to be variable in nature (Slettebak 1988) and have high rotational velocities compared to normal stars of similar spectral types. They also tend to show an excess amount of energy in the near- and far-infrared region compared to normal stars which is presumed to be due the surrounding material around the central star. Thus, the observed energy is a combination of that due to the stellar source and the surrounding material. Various attempts have been made to disentangle the stellar energy component from that of the circumstellar component in order to understand the nature, size and temperature of the envelope. These include:

a) Radius determination based on IR excess (Gehrz et al. 1974, Dachs and Hanuschik 1984; Waters et al. 1987),

b) Radius estimates from polarization and spectrophotometric data (Jones 1979),

c) Envelope dimensions derived from the width of shell absorption cores (Kogure 1969; Hirata and Kogure 1977),

d) Dachs et al. (1992) attempted to understand the physical properties, flow patterns and density distribution of the gas by a comparison of synthetic emission line profiles and empirical profiles measured for real Be stars.

Thus, the estimated disk radii are different for different authors due to the way the optical depth is defined. In this analysis, an attempt is made to estimate the photospheric temperatures, distances, the envelope size and temperature without any recourse to the definition of optical depth from the published energies in various passbands with a simple assumption that both the central star and the surrounding disk or envelope will be radiating like a blackbody and the decrement in the observed radiation strictly follows the inverse square of the distance.

\section{Data and reduction}

Published broadband magnitudes for 23 selected Be stars have been corrected for interstellar absorption and the corrected magnitudes have been converted into absolute flux units through the calibrations given by Johnson (1966) and in the IRAS Supplementary Catalogue. The observed energy distribution of each star is fitted with a representative blackbody tempera- 
ture by covering maximum number of observed energies. This temperature of the photospheric flux is thus estimated on the basis of visual inspection of the best fit between the observed and the predicted, covering as many passbands as possible while keeping in view the spectral type and luminosity classification of the star. The zero-point shift between the observed and the predicted allows us to estimate the distance to the star. Once we have established the flux emitted by the photosphere of the star in each passband through a blackbody analysis, the difference between the observed and the predicted flux could be attributed to the contribution by the circumstellar material. The distribution of this residual flux with wavelength allows us to estimate the temperature of the material. The relevant shift factor would lead to an estimation of the extension or spread of this material in terms of stellar radii. The parameters thus derived are given in Table I for all 23 stars and compared with other earlier estimates.

\section{Results}

The present analysis suggest that HD 5394, HD 22192, HD 50013, HD 63462 and HD 148184 have two component shell structure, while, HD 6811, HD 10516, HD 30076, HD 372023, HD 41335, HD 50138, HD 142983 and HD 217891 showed a single component structure. In the case of HD 20336, HD 24534, HD 35439, HD 83953, HD 86612, HD 91120, HD 120324, HD 138749 , HD 142926 and HD 217675 no predominant shell structure has been noticed except some slight excess emission at longer wavelengths in spite of that they are classified as Be stars. The present analysis suggests that our estimated shell temperatures are lower than those of Waters et al. (1987). In addition, both Waters et al. and Andrillat et al. (1990) had suggested a predominant shell structure for HD 20336 and HD 35439, while the present analysis could not confirm this. Further investigations are necessary.

This research has been carried out under grant No. SP/S2/023/89 from the Department of Science and Technology, Government of India, which is gratefully acknowledged.

\section{References}

Andrillat, A., Jaschek, M, Jaschek, C. (AJJ): 1990, Astronomy and Astrophysics 84, 11 Dachs, J., Hanuschik, R.W.: 1984, Astronomy and Astrophysics 138, 140

Dachs, J., Hummel, R.W. (DH): 1992, Astronomy and Astrophysics 95, 437

Gehrz, R.D., Hackwell, J.A., Jones, T.W.: 1974, Astrophysical Journal 191, 675

Hirita, R., Kogure, JT.L.: 1977, Publications of the ASJ 29, 477

Johnson, H.LO.: 1966, ARA\& A 4, 193

Jones, T.W.: 1979, Astrophysical Journal 228, 787

Kastner, J.A., Mazzali, P.A. (KM): 1989, Astronomy and Astrophysics 210, 295

Kogure, T.: 1969, Publications of the ASJ 27, 165

Slettebak, A.: 1988, Publications of the ASP 100, 770

Waters, L.B.F.M., Coté, J., Lamers, H.J.G.L.M. (WCL): 1987, Astronomy and Astrophysics 185, 206 


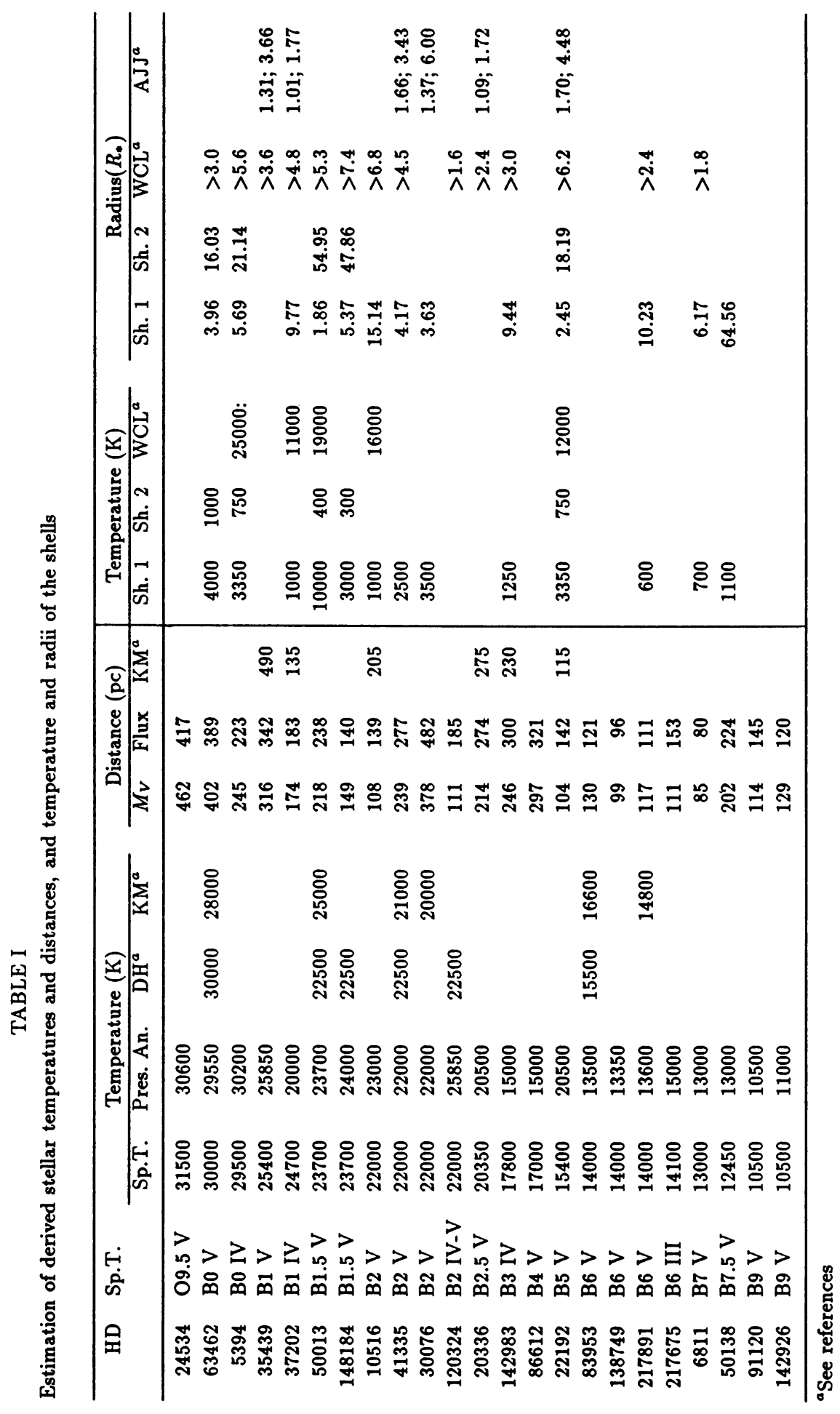

